\title{
Density dependence of in-medium nucleon-nucleon cross sections
}

\author{
C. A. Bertulani \\ Department of Physics, Brookhaven National Laboratory, \\ Upton, NY 11973-5000, USA
}

\begin{abstract}
The lowest-order correction of the density dependence of in-medium nucleonnucleon cross sections is obtained from geometrical considerations of Pauliblocking effects. As a by-product, it is shown that the medium corrections imply an $1 / E$ energy dependence of the density-dependent term.
\end{abstract}

PACS: 25.70.-z,25.75.Ld

The nucleon-nucleon cross section is a fundamental input in theoretical calculations of nucleus-nucleus collisions at intermediate and high energies $(E / A \gtrsim 100 \mathrm{MeV})$. One expects to obtain information about the nuclear equation of state by studying global collective variables in such collisions (see, e.g., [1]). Transport equations, like the BUU equation, are often used as tools for the analysis of experimental data and as a bridge to the information about the equation of state (see, e.g., [2]). The nucleon-nucleon cross sections are building blocks in these transport equations.

In previous theoretical studies of heavy ion collisions at intermediate energies $(E / A \simeq 100$ $\mathrm{MeV}$ ) the nucleon-nucleon cross section was multiplied with a constant scaling factor to account for in-medium corrections [3, [4]. As pointed out in ref. [2], this approach fails in low density nuclear matter where the in-medium cross section should approach its free-space value. A more realistic approach uses a Taylor expansion of the in-medium cross section in the density variable. One gets [5] 


$$
\sigma_{N N}=\sigma_{N N}^{f r e e}(1+\alpha \bar{\rho})
$$

where $\bar{\rho}=\rho / \rho_{0}, \rho_{0}$ is the normal nuclear density, and $\alpha$ is the logarithmic derivative of the in-medium cross section with respect to the density, taken at $\rho=0$,

$$
\alpha=\left.\rho_{0} \frac{\partial}{\partial \rho}\left(\ln \sigma_{N N}\right)\right|_{\rho=0}
$$

This parameterization is motivated by Brückner G-matrix theory and is basically due to Pauli-blocking of the cross section for collisions at intermediate energies [6]. Values of $\alpha$ between -0.4 and -0.2 yield the best agreement with involved G-matrix calculations using realistic nucleon-nucleon interactions [6].

In this article we give a simple and transparent derivation of the lowest order expansion of the in-medium nucleon-nucleon cross section in terms of the nucleon density. In our approach the leading term of the expansion comes out as $\alpha^{\prime} \rho^{2 / 3}$ with $\alpha^{\prime}$ proportional to $1 / E$. This energy-dependence agrees with experimental results on total nucleus-nucleus cross sections.

We adopt the idea that the main effect of medium corrections is due to the Pauliblocking of nucleon-nucleon scattering. Pauli-blocking prevents the nucleons to scatter into final occupied states in binary collisions between the projectile and target nucleons. This is best seen in momentum space, as shown in figure 1. We see that energy and momentum conservation, together with the Pauli principle, restrict the collision phase space to a complex geometry involving the Fermi-spheres and the scattering sphere. In this scenario, the inmedium cross section corrected by Pauli-blocking can be defined as

$$
\sigma_{N N}\left(k, K_{F 1}, K_{F 2}\right)=\int \frac{d^{3} k_{1} d^{3} k_{2}}{\left(4 \pi K_{F 1}^{3} / 3\right)\left(4 \pi K_{F 2}^{3} / 3\right)} \frac{2 q}{k} \sigma_{N N}^{\text {free }}(q) \frac{\Omega_{\text {Pauli }}}{4 \pi}
$$

where $k$ is the relative momentum per nucleon of the nucleus-nucleus collision (see figure 1 ), and $\sigma_{N N}^{f r e e}(q)$ is the free nucleon-nucleon cross section for the relative momentum $2 \mathbf{q}=$ $\mathbf{k}_{1}-\mathbf{k}_{2}-\mathbf{k}$, of a given pair of colliding nucleons. Clearly, Pauli-blocking enters through the restriction that $\left|\mathbf{k}_{1}^{\prime}\right|$ and $\left|\mathbf{k}_{2}^{\prime}\right|$ lie outside the Fermi spheres. From energy and momentum conservation in the collision, $\mathbf{q}^{\prime}$ is a vector which can only rotate around a circle with center at $\mathbf{p}=\left(\mathbf{k}_{1}-\mathbf{k}_{2}-\mathbf{k}\right) / 2$. These conditions yield an allowed scattering solid angle given by [7] 


$$
\Omega_{\text {Pauli }}=4 \pi-2\left(\Omega_{a}+\Omega_{b}-\bar{\Omega}\right),
$$

where $\Omega_{a}$ and $\Omega_{b}$ specify the excluded solid angles for each nucleon, and $\bar{\Omega}$ represents the intersection angle of $\Omega_{a}$ and $\Omega_{b}$ (see figure 1).

The solid angles $\Omega_{a}$ and $\Omega_{b}$ are easily determined. They are given by

$$
\Omega_{a}=2 \pi\left(1-\cos \theta_{a}\right), \quad \Omega_{b}=2 \pi\left(1-\cos \theta_{b}\right),
$$

where $\mathbf{q}$ and $\mathbf{p}$ were defined above, $\mathbf{b}=\mathbf{k}-\mathbf{p}$, and

$$
\cos \theta_{a}=\left(p^{2}+q^{2}-K_{F 1}^{2}\right) / 2 p q, \quad \cos \theta_{b}=\left(p^{2}+q^{2}-K_{F 2}^{2}\right) / 2 p q,
$$

The evaluation of $\bar{\Omega}$ is tedious but can be done analytically. The full calculation was done in ref. [7] and the results have been reproduced in the appendix of ref. [8]. To summarize, there are two possibilities:

$$
\begin{aligned}
& \bar{\Omega}=\Omega_{i}\left(\theta, \theta_{a}, \theta_{b}\right)+\Omega_{i}\left(\pi-\theta, \theta_{a}, \theta_{b}\right), \quad \text { for } \theta+\theta_{a}+\theta_{b}>\pi \\
& \bar{\Omega}=\Omega_{i}\left(\theta, \theta_{a}, \theta_{b}\right), \quad \text { for } \theta+\theta_{a}+\theta_{b} \leq \pi,
\end{aligned}
$$

where $\theta$ is given by

$$
\cos \theta=\left(k^{2}-p^{2}-b^{2}\right) / 2 p b .
$$

The solid angle $\Omega_{i}$ has the following values

(a) $\quad \Omega_{i}=0$, for $\theta \geq \theta_{a}+\theta_{b}$

$$
\begin{aligned}
& \Omega_{i}=2\left[\cos ^{-1}\left(\frac{\cos \theta_{b}-\cos \theta \cos \theta_{a}}{\sin \theta_{a}\left(\cos ^{2} \theta_{a}+\cos ^{2} \theta_{b}-2 \cos \theta \cos \theta_{a} \cos \theta_{b}\right)^{1 / 2}}\right)\right. \\
& +\cos ^{-1}\left(\frac{\cos \theta_{a}-\cos \theta \cos \theta_{b}}{\sin \theta_{b}\left(\cos ^{2} \theta_{a}+\cos ^{2} \theta_{b}-2 \cos \theta \cos \theta_{a} \cos \theta_{b}\right)^{1 / 2}}\right) \\
& -\cos \theta_{a} \cos ^{-1}\left(\frac{\cos \theta_{b}-\cos \theta \cos \theta_{a}}{\sin \theta \sin \theta_{a}}\right) \\
& \left.-\cos \theta_{b} \cos ^{-1}\left(\frac{\cos \theta_{a}-\cos \theta \cos \theta_{b}}{\sin \theta \sin \theta_{b}}\right)\right] \\
& \text { for }\left|\theta_{b}-\theta_{a}\right| \leq \theta \leq \theta_{a}+\theta_{b} \text {, } \\
& \text { (c) } \Omega_{i}=\Omega_{b} \text { for } \theta_{b} \leq \theta_{a}, \theta \leq\left|\theta_{b}-\theta_{a}\right| \text {, } \\
& \text { (d) } \quad \Omega_{i}=\Omega_{a} \text { for } \theta_{a} \leq \theta_{b}, \theta \leq\left|\theta_{b}-\theta_{a}\right| \text {, }
\end{aligned}
$$


The integrals over $\mathbf{k}_{1}$ and $\mathbf{k}_{2}$ in (3) reduce to a five-fold integral due to cylindrical symmetry. Two approximations can be done which greatly simplify the problem: (a) on average, the symmetric situation in which $K_{F 1}=K_{F 2} \equiv K_{F}, \mathbf{q}=\mathbf{k} / 2, \mathbf{p}=\mathbf{k} / 2$, and $\mathbf{b}=\mathbf{k} / 2$, is favored, (b) the free nucleon-nucleon cross section can be taken outside of the integral in eq. (3). Both approximations are supported by the studies of refs. [8] and can be verified numerically [7]. The assumption (a) implies that $\Omega_{a}=\Omega_{b}=\bar{\Omega}$, which can be checked using the equation (14). One gets from (四) the simple expression

$$
\Omega_{\text {Pauli }}=4 \pi-2 \Omega_{a}=4 \pi\left(1-2 \frac{K_{F}^{2}}{k^{2}}\right) .
$$

Furthermore, the assumption (b) implies that

$$
\sigma_{N N}\left(k, K_{F}\right)=\sigma_{N N}^{\text {free }}(k) \frac{\Omega_{\text {Pauli }}}{4 \pi}=\sigma_{N N}^{\text {free }}(k)\left(1-2 \frac{K_{F}^{2}}{k^{2}}\right) .
$$

The above equation shows that the in-medium nucleon-nucleon cross section is about $1 / 2$ of its free value for $k=2 K_{F}$, i.e., for $E / A \simeq 150 \mathrm{MeV}$, in agreement with the numerical results of ref. [8].

The connection with the nuclear densities is accomplished through the local density approximation, which relates the Fermi momenta to the local densities as

$$
K_{F}^{2}=\left[\frac{3 \pi^{2}}{4} \rho(r)\right]^{2 / 3}+\frac{5}{2} \xi(\nabla \rho / \rho)^{2}
$$

where $\rho(r)$ is the sum of the nucleon densities of each colliding nucleus at the position $r$. The second term is small and amounts to a surface correction, with $\xi$ of the order of 0.1 [ [].

Inserting (17) into (16), and using $E=\hbar^{2} k^{2} / 2 m_{N}$, we get (with $\bar{\rho}=\rho / \rho_{0}$ )

$$
\sigma_{N N}(E, \rho)=\sigma_{N N}^{\text {free }}(E)\left(1+\alpha^{\prime} \bar{\rho}^{2 / 3}\right) \quad \text { where } \quad \alpha^{\prime}=-\frac{48.4}{E(M e V)}
$$

where the second term of (17) has been neglected. This equation shows that the local density approximation leads to a density dependence proportional to $\bar{\rho}^{2 / 3}$. The Pauli principle yields a $1 / E$ dependence on the bombarding energy. This behavior arises from a larger phase space available for nucleon-nucleon scattering with increasing energy. 
The nucleon-nucleon cross section at $E / A \lesssim 200 \mathrm{MeV}$ decreases with $E$ approximately as $1 / E$. We thus expect that, in nucleus-nucleus collisions, this energy dependence is flattened by the Pauli correction, i.e., the in-medium nucleon-nucleon cross section is more flat as a function of $E$, for $E \lesssim 200$, than the free cross section. For higher values of $E$ the Pauli blocking is less important and the free and in-medium nucleon-nucleon cross sections are approximately equal. These conclusions are in agreement with the experimental data for nucleus-nucleus reaction cross sections [9]. This was in fact well explained in ref. [8].

Notice that, for $E / A=100-200 \mathrm{MeV}$, and $\rho \simeq \rho_{0}$, eq. (18) yields a coefficient $\alpha^{\prime}$ between -0.2 and -0.5 . This is in excellent agreement with the findings based on the BUU calculations, primarily intended to reproduce the experimental data on collective variables in intermediate energy nucleus-nucleus collisions.

In conclusion, we have presented a microscopic derivation of the lowest order density correction for the in-medium nucleon-nucleon cross section. Despite its simplicity, the calculation shows that Pauli-blocking is able to explain almost entirely the magnitude of the correction term, although the power of density dependent term is slightly different from what is commonly mentioned in the literature [2 6]. We also predict an energy dependence of the in-medium cross sections which was not accounted for previously. This calls for a further study of the consequence of this energy dependence in the transport equation analysis of collective variables in nucleus-nucleus collisions at intermediate energies.

\section{Acknowledgments}

The author is a fellow of the John Simon Guggenheim foundation. This work has been authored under Contract No. DE-AC02-98CH10886 with the U.S. Department of Energy, and partial support from the Brazilian funding agency MCT/FINEP/CNPQ(PRONEX), under contract No. 41.96.0886.00, is also acknowledged.

\section{Figure Captions}

1. The geometric description of Pauli blocking, in momentum space, for binary collisions 
of target and projectile nucleons. 


\section{REFERENCES}

[1] H.H. Gutbrot, A.M. Poskanger, and H.G. Ritter, Rep. Prog. Phys. 52, 1267 (1989)

[2] G.D. Westfall, W. Bauer, D. Craig, M. Cronqvist, E. Gualtieri, S. Hannuschke, D. Klakow, T. Li, T. Reposeur, A.M. Vander Molen, W.K. Wilson, J.S. Winfield, J. Yee, S.J. Yennello, R. Lacey, A. Elmaani, J. Lauret, A. Nadasen and E. Norbeck., Phys. Rev. Lett. 71, 1986 (1993)

[3] G.F. Bertsch, W.G. Lynch, and M.B. Tsang, Phys. Lett. B189, 738 (1987)

[4] W. Bauer, Phys. Rev. Lett. 61, 2534 (1988); W. Bauer, C.K. Gelbke, and S. Pratt, Annu. Rev. Nucl. Part. Sci. 42, 77 (1992)

[5] D. Klakow, G. Welke, and W. Bauer, Phys. Rev. C 48, 1982 (1993)

[6] T. Alm, G. Röpke, W. Bauer, F. Daffin and M. Schmidt, Nucl. Phys. A587, 815 (1995); A. Schnell, G. Röpke, U. Lombardo, and H.J. Schulze, Phys. Rev. C 57, 806 (1998)

[7] C.A. Bertulani, Braz. J. Phys. 16, 380 (1986);

[8] M.S. Hussein, R.A. Rego, and C.A. Bertulani, Phys. Reports 201, 279 (1991)

[9] S. Kox, A. Gamp, C. Perrin, J. Arvieux, R. Bertholet, J.F. Bruandet, M. Buenerd, Y. El Masri, N. Longequeue and F. Merchez, Phys. Lett. B159, (1985) 15 

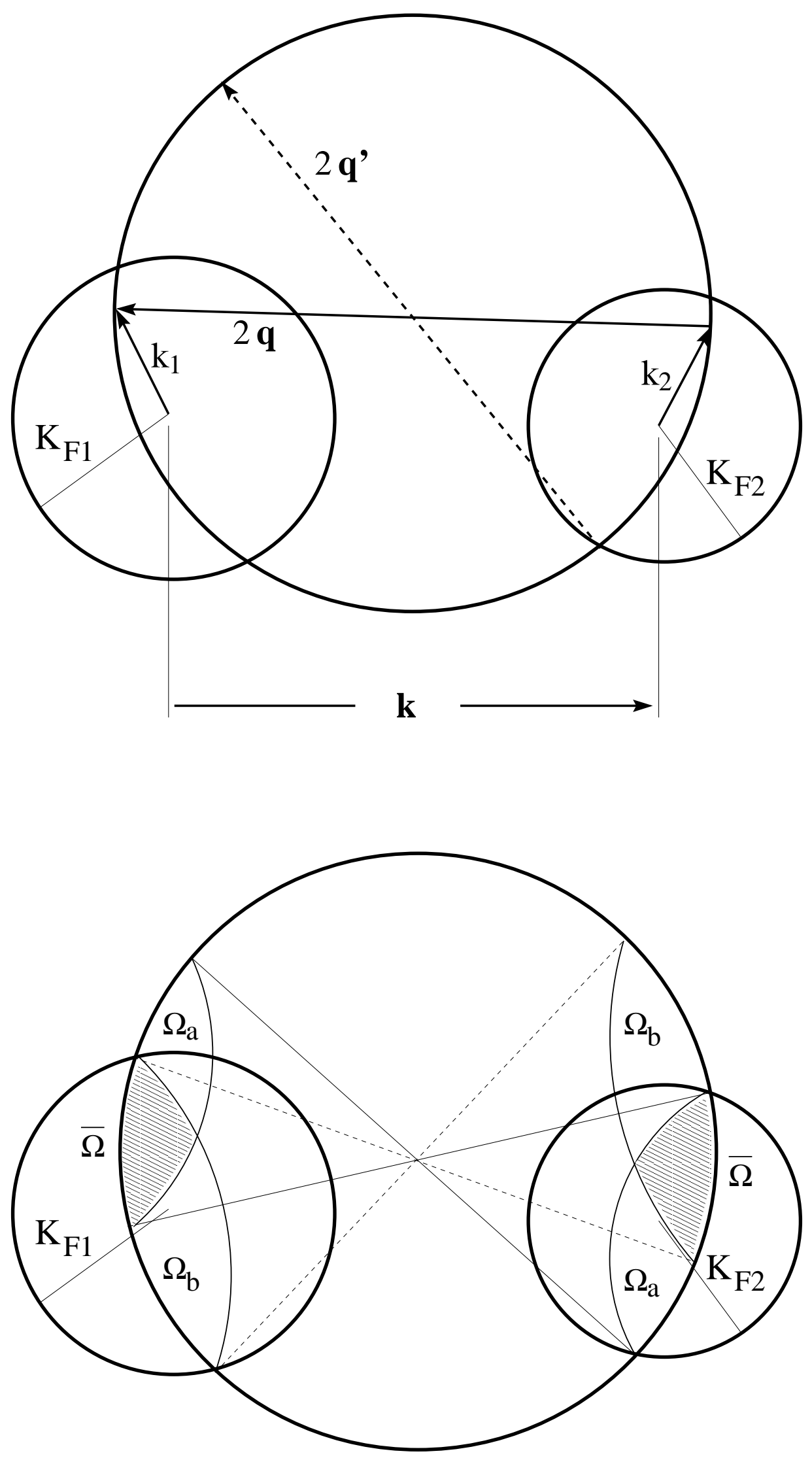